\title{
The role of dynamic information in the recognition of unfamiliar faces
}

\author{
FIONA CHRISTIE and VICKI BRUCE \\ University of Stirling, Stirling, Scotland
}

\begin{abstract}
The effects of movement on unfamiliar face recognition were investigated. In an incidental learning task, faces were studied either as computer-animated (moving) displays or as a series of static images, with identical numbers of frames shown for each. The movements were either nonrigid transformations (changes in expression) or rigid rotations in depth (nodding or shaking). At test, participants saw either single, static images or moving sequences. Only one experiment showed a significant effect of study type, in favor of static instances. There was no additional advantage from studying faces in motion in these experiments, in which both study types showed the same amounts of information. Recognition memory was relatively unaffected by changes in expression between study and test. Effects of viewpoint change were large when expressive transformations had been studied but much smaller when rigid rotations in depth had been studied. The series of experiments did reveal a slight advantage for testing memory with moving compared with static faces, consistent with recent findings using familiar faces. Future work will need to examine whether such effects may also be due to the additional information provided by an animated sequence.
\end{abstract}

When we look at a face, with little apparent effort we are able to extract a wide variety of information from it, such as gender, emotional expression, and, for familiar faces, identity. Several models have been proposed that attempt to break down the processing of such information into discrete functions (see, e.g., Bruce \& Young, 1986). The series of experiments described in this paper examines representational processes underlying the recognition of previously unfamiliar faces. According to the Bruce and Young model, facial recognition relies on being able to arrive at a structural representation. Recognition occurs when what we see successfully "matches" (or is judged to be a legitimate variation on) what we have "stored." The aim of this paper was to investigate whether movement helps to build, and/or retrieve, a representation of a previously unfamiliar face.

The typical research paradigm used to investigate the recognition of unfamiliar faces presents participants with single pictures of previously unfamiliar faces and examines how well these faces can be recognized when later shown in the same or a different picture from that studied (see Shapiro \& Penrod, 1986, for an overview and metaanalysis of many such studies). Such studies usually show high rates of recognition memory when the test pictures match those studied, but much lower accuracy when memory is probed with a different picture of the studied individual. For example, Bruce (1982) showed that the hit rate for identical pictures of unfamiliar faces was $90 \%$, but when both the facial expression and viewpoint were

F.C. is now at the University of Paisley. Correspondence should be addressed to V. Bruce, Department of Psychology, University of Stirling, Stirling FK9 4LA, Scotland (e-mail: vicki.bruce@stir.ac.uk).

-Accepted by previous editor, Geoffrey R. Loftus changed at test, the hit rate dropped to $61 \%$, even though many cues to identity, such as hairstyle, facial hair, and clothing, remained available. Such studies suggest that people are remarkably poor at encoding a representation from a single picture of a face that allows extrapolation to a novel viewpoint or expression.

Despite the limitations observed in experiments of this design, in everyday life we are generally able to deal with a range of variations in viewpoint, lighting, expression, and (within limits) hairstyle when recognizing faces with which we are already familiar. In order to recognize faces in this way, we need either to store enough instances from which to generalize to novel ones, or we must store faces in some other way that enables generalization to occur, perhaps by extracting and storing information that is invariant across different views and expressions. Earlier studies of object recognition emphasized the storage of viewpointinvariant information in the form of 3-D models (e.g., Biederman, 1987; Marr \& Nishihara, 1978); more recently, research has postulated that (at least for certain types of objects) viewpoint-specific information is stored (e.g., Tarr \& Bulthoff, 1995). Thus approaches to object recognition include both 3-D model-based \& exemplar-based representational theories. However, few objects undergo the sorts of continuous, nonrigid changes that are characteristic of human faces as they speak and express, and there has been little consideration of how this sort of variation may be stored or processed in face recognition.

Several authors have questioned the paradigm normally applied to experiments investigating face recognition, which tends to favor the instance-based approach; moreover, picture recognition is confounded with true face recognition when participants are shown a photograph of an unfamiliar face and later tested with the same or a dif- 
ferent photograph (e.g., Bartlett \& Leslie, 1986; Bruce, 1982; Vokey \& Read, 1992). Klatzky and Forrest (1984) stated that the use of only a single photograph during presentation would encourage the coding and memory of a view-specific episode, which would then form the basis for later old-new discrimination by the participant. In order to investigate how representations of unfamiliar faces may more typically be encoded and accessed, we need to study situations more similar to those of everyday life, where faces are usually seen in dynamic sequences provided by movements of the faces themselves and by the observer.

There are several possible ways in which studying a dynamic sequence might be expected to build a more robust representation of the face. First, when we see a moving sequence, we sample more "instances" (individual viewpoints, expressions, etc.), thereby providing a larger set of exemplars to be stored for an individual face. Second, according to Ullman's (1979) algorithm for inferring form from motion, moving sequences should provide a better 3-D representation of that face, which could enhance our ability to recognize that person in a previously unseen viewpoint. Third, moving sequences should provide a better range of variation from which to extract any other kinds of invariant characteristics of faces, (i.e., aspects that remain constant over changes in viewpoint and expression).

For similar reasons, we might also expect benefits when moving sequences are used to probe memory for faces, regardless of how these were initially studied. For example, if memory for the face is tested in a pose different from that originally studied, a dynamic sequence should provide a greater range of exemplars and thus a greater chance of generalizing from that originally studied. If dynamic sequences furnish better 3-D representations or promote better extraction of invariants, this should also lead to benefits if memory is probed in this manner.

Consistent with these suggestions, the literature on face recognition does contain several examples of investigations showing advantages when dynamic sequences are used either at study or to test face recognition. Pike, Kemp, Towell, and Phillips (1997) compared learning of faces from a moving sequence with those learned in a series of static pictures. They used faces illuminated by a single light source and filmed from a fixed point while the actors were seated in a rotating chair. The learning phase in their first two experiments presented a single static (full-face) image, five static images of the head turning, or a dynamic sequence in which the target was shown on a video with a full $360^{\circ}$ horizontal rotation of the chair. The test was of a single (full-face) static image, different from any shown in the learning sequence. Pike et al. found a significant advantage for faces learned via a coherent moving sequence, with those faces learned via multiple static views providing the next best performance. In further experiments the advantage of studying moving sequences was maintained even when the comparison was with a multistatic condition with 10 distinct viewpoints.
Bruce and Valentine (1988) reported a similar, though nonsignificant, trend in an experiment in which study was of a single image, multiple images, or a video film, and in which test images were single images shown from a different viewpoint and photographed on a different occasion from those studied.

Another experimental method used to investigate face recognition is one that attempts to replicate the situation of being an eyewitness to a crime; presentation of a single face (of the "criminal") requires later identification from a series of distractors. Using such a method, Shepherd, Ellis, and Davies (1982) found no advantage for a learning phase featuring a moving video sequence over a condition that used a single static photo for study. However, they did find that participants who initially saw the "target" in person had better subsequent recognition rates than those seeing either video or static presentations. They attributed this to different strategies that people employed in looking at "live" faces, as opposed to filmed or photographed faces; this finding may reflect the type of incidental learning task they used, in which during the learning phase, participants were asked to assess the honesty of the target person. This illustrates the complexity of additional processes that may come into play when witnesses observe or interact with a complex incident, which may involve very much more than memory for the face alone.

Nonetheless, using a similar eyewitness methodology, Schiff, Banka, and de Bordes Galai (1986) did find an advantage for testing unfamiliar face recognition using a moving sequence, rather than a static mug shot. They found that the level of similarity between learning and testing formats did not fully account for differences in recognition accuracy in their experimental conditions. Performance was best in the condition in which participants witnessed a dynamic event and were tested with a dynamic sequence (which showed a moving $180^{\circ}$ sweep of the camera around the face). These recognition rates were higher than those in any of the conditions in which participants saw the "event" and were then tested with either a freeze-frame taken from the same event, or a later mug shot.

Perhaps the most convincing evidence about the advantageous contribution of movement to face recognition comes from the recent work of Knight and Johnston (1997). They showed that motion helps in the recognition of famous faces when these were presented in photographic negatives. Negative moving sequences were recognized very much more accurately than single negative images. A related effect was reported by Bruce and Valentine (1988). When faces that were personally familiar to observers were presented as a dynamic point-light display of 100 white dots (see Bassili, 1978), recognition of individual identity was significantly better when the display was shown in motion than when it was shown statically, with the static condition yielding recognition performance no better than chance. These experiments thus suggest that recognition of familiar faces (which will have 
previously been seen in both dynamic and static forms) presented in difficult circumstances can be enhanced when the test is dynamic, and the authors of both studies offered explanations of the source of this benefit coming from the provision of dynamic 3-D structure-from-motion information.

So, although advantages from the use of dynamic sequences have not always been found in the literature, a number of studies have revealed beneficial effects of dynamic sequences at study (e.g., Pike et al., 1997), or in some combination of study and test (e.g., Bruce \& Valentine, 1988; Knight \& Johnston, 1997; Schiff et al., 1986). However, a fundamental problem is that we need to see only one frame to perceive a static image; a prerequisite of the perception of motion is that more than one frame is presented ( $1 \mathrm{sec}$ of a moving video sequence contains between 25 and 50 frames). Therefore, it may be the case that movement in either phase of an experiment is advantageous purely because it contains more information than a static presentation, and that coherent movement per se adds little to perceptual processing. To examine why moving sequences may benefit recognition, we need to examine whether benefits are found when information content is equated.

The series of four experiments presented here aimed to compare recognition of faces that were studied using moving or static sequences, with equal amounts of information contained in each, by showing identical numbers of frames. In different experiments, the faces were learned carrying out both rigid and nonrigid (expressive) movements.

The results of this investigation should help us to understand the nature of the representations that mediate the recognition of previously unfamiliar faces. If faces are represented as collections of instances, then when the information content of the two conditions is equated, there should be no benefit for studying dynamic sequences. However, if recognition of faces in novel viewpoints or expressions is facilitated by the extraction of a 3-D modelbased representation, dynamic conditions favoring the extraction of 3-D structure from motion should enhance recognition performance. Our experimental hypothesis was therefore that participants should be able to build up a better representation of an unfamiliar face if it was learned in a dynamic display rather than a static one. However, because a mismatch between the format studied and tested could impede recognition, we further examined whether any benefits that arose from the study of dynamic sequences might be revealed only when the test phase also probed memory using dynamic sequences. Thus, in all four experiments we compared memory for faces studied in static and dynamic sequences, but Experiments $1 \mathrm{~A}$ and $2 \mathrm{~A}$ used static images at test, and Experiments $1 \mathrm{~B}$ and 2B used dynamic sequences at test. In all experiments, we compared recognition memory for faces tested in different viewpoints from those originally studied, with that shown for faces retested in the same viewpoint.
As indicated earlier, faces move both nonrigidly, when speaking and expressing emotions, and rigidly, through rotations in depth. The mechanisms by which representations are constructed across expressive variations are not necessarily the same as those used to achieve recognition across changes in viewpoint. Bruce and Young (1986), influenced by the viewpoint specificity shown by monkey inferotemporal neurones selective for faces (see, e.g., Perrett et al., 1985), argued that structural representations for face recognition might be viewpoint dependent, but expression independent. Consistent with this, Bruce (1994) discussed how variations in appearance within the same viewpoint, occurring when a face makes expressive movements, seem to be treated in memory in a way that favors the prototype - or average - of the series encountered, whereas between-view variations do not give rise to such prototype effects. Cabeza, Bruce, Kato, and Oda (in press) have provided further evidence for this position. It is possible, then, that variations in viewpoint are treated as discrete instances whereas variations in expression are not, and that presentation of dynamic sequences may have different consequences for recognizing faces in novel expressions compared with novel viewpoints. In Experiments 1A and 1B, participants saw faces in expressive sequences (i.e., movement of internal features), and we examined the accuracy of recognition when the expression was maintained or changed at test, as well as manipulating viewpoint. In Experiments $2 \mathrm{~A}$ and $2 \mathrm{~B}$, the presented faces made rigid (head-turning) gestures, and we examined recognition rates when the gesture was the same or different at test.

\section{GENERAL METHOD}

\section{Materials}

Target and distractor actors were filmed against a black background, wearing a bathing cap, and with a towel wrapped around their shoulders. This was to eliminate any subsequent cues to recognition being derived from clothing or hairstyle and to focus attention on the internal features and shape of the face. The actors carried out a variety of facial gestures and head movements, including the changes in configuration associated with a continuum from a smile to a sad gesture (the person had to raise and then lower the corners of the mouth); the actors performed the mouth movements while pronouncing the speech sound "eeh," followed immediately by the "ooh" sound; they were also filmed nodding and shaking their heads. Actors were filmed simultaneously from two perspectives: full-face $(\mathrm{FF})$ shots and three-quarter $(\mathrm{TQ})$ profile views. In the TQ view, the right side of the face was filmed at an angle so that there was a clear view of the right side of the face; the left eye was visible up to the midpoint of the eyebrow; the right half of the left eye was also visible, as was the bow of the lips. The two cameras used were Sony Super-8s, and the actor was seated approximately $45 \mathrm{~cm}$ away from each camera.

Frames were grabbed from the films using the Quick Image package, which allowed for the selection of specific frames from each of the required gestures. The TQ frames were chosen to show the same points of articulation (or rotation) as each of the FF frames. Since the purpose of the experiments was to ascertain the benefits of movement per se, over and above the amount of information embedded in the sequences, it was essential to have an economical set 
of frames that could run together, encapsulating a moving face. It was also important to have a small number of frames so that the animation package (Xrastool) could show all target learning phases in one entire film. Several attempts were made to find the smallest number of frames that would allow for the perception of coherent "natural" movement of the face or head when played at $150 \mathrm{msec}$ per frame (using the Xrastool application). Five independent observers judged that, for internal expression changes, five frames were adequate; three were required for the changes in angle/rotational displacements (see Method section for Experiments $2 \mathrm{~A}$ and $2 \mathrm{~B}$ for further details).

Each sequence of frames was stored as 8-bit gray scale images, $156 \times 156$ pixels. Using Xrastool on a Sun Workstation, the physical size of each frame (on the screen) was $7.5 \times 7.5 \mathrm{~cm}$ for each experiment's presentation phase, and also for the test phases in Experiments $1 B$ and $2 B$. The frame size for the test phases of Experiments $1 \mathrm{~A}$ and $2 \mathrm{~A}$ using Superlab (on a Macintosh Centris 650) was $9 \times 9.5 \mathrm{~cm}$.

\section{Moving Versus Static Presentation}

The same principles applied to the manufacture of each type of stimulus for all four experimental study and test phases, but see individual experiments for details.

In Experiments $1 \mathrm{~A}$ and $1 \mathrm{~B}$, for the faces that were to be shown moving in the study phase, five FF frames depicting the smile-sad gesture were selected. These were played through Xrastool at a rate of $150 \mathrm{msec}$ per frame on the Sun Workstation. Each target was randomly allocated to a position in the learning "film." Each actor's sequence was shown four times in correct running order (with the effect of that person making a smooth facial gesture), giving a total of 20 frames per gesture. There were two blank, black frames shown at the beginning and end of each of these blocks of 20 frames (the reason for these blank frames becomes important for the static sequences, described below). The running order for the "moving" presentation of each target was therefore blank, blank, 12345, $12345,12345,12345,12345$, blank, blank (frame 1 denotes the numerical start of the sequence, but the initial articulatory point of the gesture was randomized for each target). The screen was then blank (gray) for $2 \mathrm{sec}$ between each stimulus sequence, (achieved by inserting a gray frame for $2 \mathrm{sec}$ in the Xrastool film "script"): This was for participants to describe the face aloud to the experimenter (see below).

For faces to be shown in the static presentation format, the same set of five frames just described was shown in a predetermined random sequence to eliminate any perception of apparent motion between each set (i.e., it was not a coherently ordered presentation). Each separate frame was played four times in succession so that the sequence (when animated using Xrastool at $150 \mathrm{msec}$ per frame) would be experienced as a cycle of static pictures. One of the blank, black frames used at the beginning of the moving sequences was used to separate each static "block" of four. This ensured that participants were exposed to the same amount of information (identical number of frames) as they were in the moving presentation format, and also that each one would be seen for the same duration. The starting point of each target learning sequence was (again) randomized to eliminate any effects of initial exposure and learning. The running order of frames for the static study phase could be, for example: 2222, blank, 5555, blank, 3333, blank, 1111 , blank, 4444 .

\section{Presentation Counterbalancing}

In all four experiments, there were 5 male and 5 female target faces: Of these, 5 were learned by half the participants in each condition in an animated (moving) sequence, and 5 were learned in a static sequence. All faces changed their moving/static status for the remaining subgroups of participants. Of the 5 males shown to one subgroup, 2 were moving and 3 were static: of the 5 females shown to the same subgroup, 3 were moving and 2 were static. The order in which the faces appeared in the film was randomly selected, but maintained for subgroups of participants.

\section{General Design}

The experiments are described in pairs (1A and $1 \mathrm{~B}, 2 \mathrm{~A}$ and $2 \mathrm{~B}$ ). The $A$ and $B$ versions of each experiment differ in the format used for the test phase-static images (A) versus dynamic sequences (B). However, these were conducted as distinct experiments, with some variation in the size and nature of the participant populations sampled, and differences in the machines on which tests were conducted and the way in which responses were recorded; therefore, experiments within a pair have been analyzed separately. In each experiment, participants were randomly assigned to one of four between-subjects conditions.

The design in each experiment was a $2 \times 2 \times 2$ factorial. The within-subjects factor in all experiments was presentation format (static or dynamic). All participants initially studied half the faces in dynamic and half in static formats. Each experiment had two further between-subjects factors. One of these varied the viewpoint/ plane in which the face was tested-either the same or different from that studied. The other factor varied was the expression/gesture. In Experiments $1 \mathrm{~A}$ and $1 \mathrm{~B}$, the learning gesture was always a smile-sad expressive sequence, and the test gesture was either the same or changed to show a facial speech movement. In Experiments $2 \mathrm{~A}$ and $2 \mathrm{~B}$, nodding and shaking head gestures were shown to different groups of participants in learning and test phases.

\section{General Procedure}

Study phases. All experiments tested incidental memory for faces. In all presentation phases, participants were required to judge whether each of the faces shown was an "arts" or a "science" student. Participants were told that some faces would be seen in animated sequences, some as a series of stills, for about $3 \mathrm{sec}$ each. Following each face, there would be a blank screen for $2 \mathrm{sec}$, during which they were to give their decision aloud, for the experimenter to write down. They then carried out a series of unrelated filler tasks for approximately $30 \mathrm{~min}$, before completing the recognition task.

Test phases. The test phases of Experiments $1 \mathrm{~A}$ and $2 \mathrm{~A}$ required participants to make their recognition decisions on the basis of single static frames: These depicted the apex of the relevant facial, or rotational, gesture (see individual experiments). In both cases, Superlab was used to measure the accuracy and latencies for recognition decisions for the 10 targets. The faces of the targets were interleaved within a series of faces that included 10 distractors, and these were presented in a different random order for each participant

The test phases of Experiments $1 \mathrm{~B}$ and $2 \mathrm{~B}$ involved participants making recognition decisions verbally to moving sequences, tested on the same Sun Workstation as that used for presentation, and response latencies were not recorded for these experiments. To create these test sequences, two Xrastool movies were made for each experiment, using the same procedure as that employed in the learning phase, with targets and distractors being randomly allocated to either film. In each case, there were 6 target faces randomly interleaved with 4 distractors in the first test film, and 4 targets with 6 distractors in the second. Participants were informed that each target was represented only once in either of the two test films.

\section{Treatment of Results}

Although latencies as well as accuracies were recorded in two of the four experiments, the analyses of accuracies only are presented here because performance was highly error prone. Participants' performance was examined using separate analysis of hits and false positives (FPs), and also using $\mathrm{A}^{\prime}$ and $\mathrm{B}^{\prime \prime} . \mathrm{A}^{\prime}$ is the nonparametric discrimination index (the participant's sensitivity to the signal), where chance is $\mathrm{A}^{\prime}=.5 . \mathrm{B}^{\prime \prime}$ is the nonparametric bias index, which varies between -1 and $+1: \mathrm{B}^{\prime \prime}=0$ shows a neutral criterion, $\mathrm{B}^{\prime \prime}>$ 0 a liberal criterion, and $\mathrm{B}^{\prime \prime}<0$ shows a conservative (cautious) cri- 
Table 1

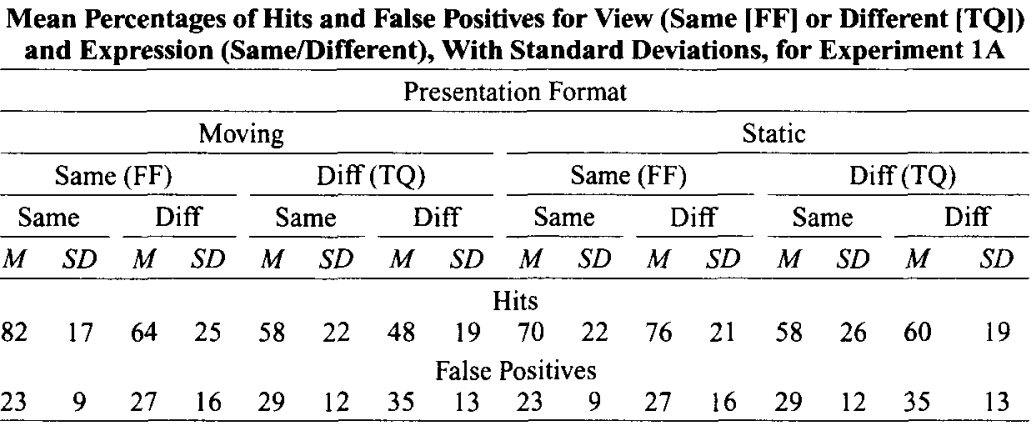

Note-FF, full-face view; TQ, three-quarters view.

terion (see Snodgrass \& Corwin, 1988). Calculated mean B" values in the conditions tested in these experiments ranged between -0.11 and +0.08 (i.e., showing generally neutral bias), and since no significant effects of $B$ " were found in any of the four experiments, we do not report these analyses.

Hit rates were entered into $2 \times 2 \times 2$ factor analysis of variance (ANOVAs). FPs were entered into $2 \times 2$ factor ANOVAs, corresponding to the between-subjects factors, which were varied. Since the distractors were seen only at test, the FP rate could be measured only for the specific test format used (i.e., static images in Experiments $1 \mathrm{~A}$ and $2 \mathrm{~A}$; dynamic sequences in Experiments $1 \mathrm{~B}$ and $2 \mathrm{~B}$ ). To compute $A^{\prime}$ and $B^{\prime \prime}$ measures, the single FP rate for each of the four test cells was applied to both presentation formats. Items analyses were also carried out on hit rates to check for the generality of the effects across different targets. These are reported only when there is a difference from the by-subjects analyses. Because of the small numbers of targets, the items analyses lack power, but nonetheless they generally confirm the statistically significant effects found in the by-subjects analyses. To simplify the results sections here, we focus on the $A^{\prime}$ analyses, but note where any of the other analyses yielded discrepant results. Tables of hits and false positives are provided for each experiment, as well as graphs of $A^{\prime}$.

\section{EXPERIMENTS 1A AND 1B}

In both experiments, the study sequence $\mathrm{FF}$ images showed an expressive movement, the smile-sad gesture. The test sequence showed still images in Experiment 1A and dynamic sequences in $1 \mathrm{~B}$.

\section{Method}

Participants. There were 40 participants in Experiment 1A: 19 males and 21 females between 18 and 30 years. They were students and staff at University of Stirling. Ten participants were randomly allocated to each of four conditions. There were 32 student participants in Experiment 1B: 16 male and 16 female, with 8 in each of the four conditions.

Design. In Experiments 1A and 1B, the four between-subjects test conditions were constructed from all combinations of the two between-subjects factors of viewpoint at test (either FF or TQ view) and expression at test (either the same or changed from that studied). In Condition 1, FF-same expression, faces were tested from the (studied) FF viewpoint, posing the same facial expression as that studied initially (a smile). Thus, in Experiment 1A, the static test images exactly matched those images studied for half the targets. In Experiment IB, the moving test images exactly matched the dynamic sequences studied for half the targets. In Condition 2, TQ-same expression, faces were tested from the TQ viewpoint, posing the same expression as at study (a smile). In Condition 3,
FF-different expression, faces were tested from the (studied) FF viewpoint, posing a different expression to that in the presentation phase. In Experiment 1A, the test phase used static targets and distractors at the apex of the "ooh" speech sound. In Experiment 1B, the dynamic sequences showed the face varying from an "eeh" to an "ooh" speech sound. In Condition 4, TQ-different expression, faces were tested from the TQ viewpoint, posing the "ooh" speech sound.

Procedure. The study phase was as described. For the test phases, in Experiment 1A, the test series of 20 static images ( 10 targets and 10 distractors) was presented in a random order, in one of the conditions outlined. Participants' decisions were indicated by a buttonpress. Participants in the various conditions were told about how the faces would appear in their particular test phase. They were also informed that they should press the corresponding button as soon as they had reached their recognition decision and that the face would be on the screen only for as long as it took them to make their decision. There was a 2-sec interval between their response and the next test face.

In Experiment 1B, each of the 20 test sequences was seen in a previously assigned random order. Each face was seen for $3 \mathrm{sec}$ (five frames $\times 150$ msec per frame $\times$ four repeats), as in the dynamic study sequences. Participants were informed of the nature of their test (according to their assigned condition) and that they were to give recognition decisions verbally, for the experimenter to record.

\section{Results of Experiment 1A}

The mean hit rate across all conditions was $64 \%$ and the FP rate was $28 \%$. Figure 1 plots mean $\mathrm{A}^{\prime}$ in each condition of interest. An ANOVA of the $A^{\prime}$ values calculated for each participant demonstrated a significant effect of viewpoint, with better discrimination when the faces were tested in FF views $[F(1,36)=18.6, p<.01]$ and a significant interaction between expression and presentation $[F(1,36)=5.84, p<.05]$, which reflected a (nonsignificant) tendency for faces studied moving to show a drop in performance when expression was changed. There were no other main effects or interactions (all $p s>.1$ ) in the $\mathrm{A}^{\prime}$ analysis. Analyses of hits showed only the significant main effect of viewpoint, whereas analysis of FPs showed no significant effects (see Table 1 for mean hits and FPs in each condition).

\section{Results of Experiment 1B}

The mean overall hit rate was $64 \%$; the FP rate was $28 \%$. Figure 2 plots mean $\mathrm{A}^{\prime}$ for each condition. ANOVA of $A^{\prime}$ scores for each participant showed a significant effect only of viewpoint $[F(1,28)=18.16, p<.01]$. Dis- 


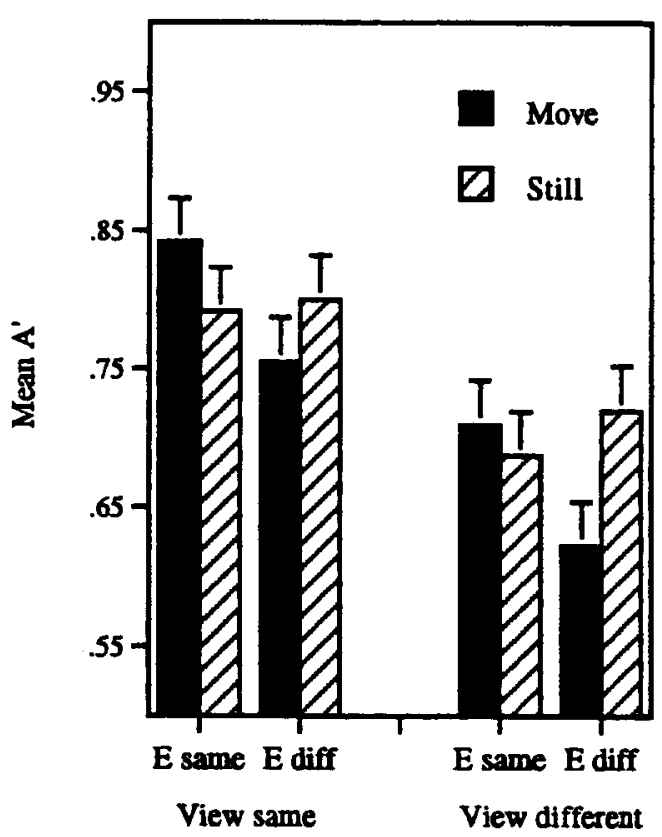

Figure 1. Mean $A^{\prime}$ in each condition of Experiment $1 A$ (static test). The error bars show the $95 \%$ confidence interval for the within-subjects comparison (Loftus \& Masson, 1994) between presentation conditions (move vs. still) as a function of expression (E, same or different) and viewpoint (same or different).

crimination was again better when faces were tested in FF views. No other main effects or interactions reached significance (all $p \mathbf{s}>.1$ ). Analyses of hit rates was consistent with the $A^{\prime}$ analysis, whereas analysis of FPs yielded no significant effects (see Table 2 for the hits and FPs in each condition).

\section{Discussion}

Neither Experiment 1A nor 1B revealed any significant overall difference between the moving and static presentation sequences: In Experiment 1A, slightly more faces were recognized from the series of static images $(63 \%$ mean hits for faces studied moving compared with $66 \%$ for those studied as static). The use of static images in the test phase is likely to favor the static presentation format given the general benefit to memory when study and test conditions match (see, e.g., Davies, Ellis, \& Shepherd, 1978; Tulving \& Thomson, 1983). Using dynamic test sequences in Experiment 1B led to the reversal of this slight overall trend (hit rates of $66 \%$ for faces studied moving compared with $63 \%$ for those studied static).

Both experiments revealed a highly significant effect of changing view between study and test, with fewer hits and a tendency for higher FPs in the TQ conditions, but no significant overall effect of changing expression. Participants were better able to generalize across differences within the same plane (i.e., to a different expression) than they were able to generalize across differences between planes of viewing and test (and the significant interaction in the $\mathrm{A}^{\prime}$ analysis of Experiment $1 \mathrm{~A}$ indicated that this generalization was somewhat less effective when moving images had been studied). This is in accordance with the findings of Bruce (1994) and Cabeza et al. (in press), who used a quite different prototype-learning paradigm to show that various viewpoints appear to be stored separately in face memory. This is also consistent with Bruce and Young's (1986) suggestion that the structural codes mediating face recognition are viewpoint dependent but independent of expression. However, it may be that the effects of changing expression were modest because the learning sequences showed sufficient variation in the smile-sad gesture (with intermediate stages within that transition), to allow for generalization to a different facial expression. It is also possible that performance with a change in view was poor because no variation in viewpoint was experienced. Experiments $2 \mathrm{~A}$ and $2 \mathrm{~B}$ allowed us to examine whether generalization to novel viewpoints is better when variations in head angle are seen at study.

\section{EXPERIMENTS 2A AND 2B}

In Experiments 2A and 2B, the emphasis was on rigid transformations of the head. Two of the variables under manipulation at test were similar to those in Experiments $1 \mathrm{~A}$ and $1 \mathrm{~B}$ - the effect of presentation format (either moving or static) and the effect of changing viewpoint between study and test, which in these experiments we label "plane," since it corresponds to the plane in which the head rotates. When the head shakes, as in disapproval, the face is seen in TQ and profile for part of the time, and

Table 2

Mean Percentages Hits and False Positives for View (Same [FF] or Different [TQ]) and Expression (Same/Different), With Standard Deviations, for Experiment $1 B$

\begin{tabular}{|c|c|c|c|c|c|c|c|c|c|c|c|c|c|c|c|}
\hline \multicolumn{16}{|c|}{ Presentation Format } \\
\hline \multicolumn{8}{|c|}{ Moving } & \multicolumn{8}{|c|}{ Static } \\
\hline \multicolumn{4}{|c|}{ Same (FF) } & \multicolumn{4}{|c|}{$\operatorname{Diff}(T Q)$} & \multicolumn{4}{|c|}{ Same (FF) } & \multicolumn{4}{|c|}{$\operatorname{Diff}(\mathrm{TQ})$} \\
\hline \multicolumn{2}{|c|}{ Same } & \multicolumn{2}{|c|}{ Diff } & \multicolumn{2}{|c|}{ Same } & \multicolumn{2}{|c|}{ Diff } & \multicolumn{2}{|c|}{ Same } & \multicolumn{2}{|c|}{ Diff } & \multicolumn{2}{|c|}{ Same } & \multicolumn{2}{|c|}{ Diff } \\
\hline$M$ & $S D$ & $M$ & $S D$ & $M$ & $S D$ & $M$ & $S D$ & $M$ & $S D$ & $\bar{M}$ & $S D$ & $M$ & $S D$ & $M$ & $S D$ \\
\hline \multicolumn{16}{|c|}{ Hits } \\
\hline 85 & 14 & 75 & 18 & 50 & 19 & 53 & 21 & 77 & 23 & 75 & 21 & 50 & 21 & 50 & 19 \\
\hline \multicolumn{16}{|c|}{ False Positives } \\
\hline 20 & 23 & 30 & 9 & 29 & 12 & 34 & 19 & 20 & 23 & 30 & 9 & 29 & 12 & 34 & 19 \\
\hline
\end{tabular}

Note--FF, full-face view; TQ, three-quarters view. 


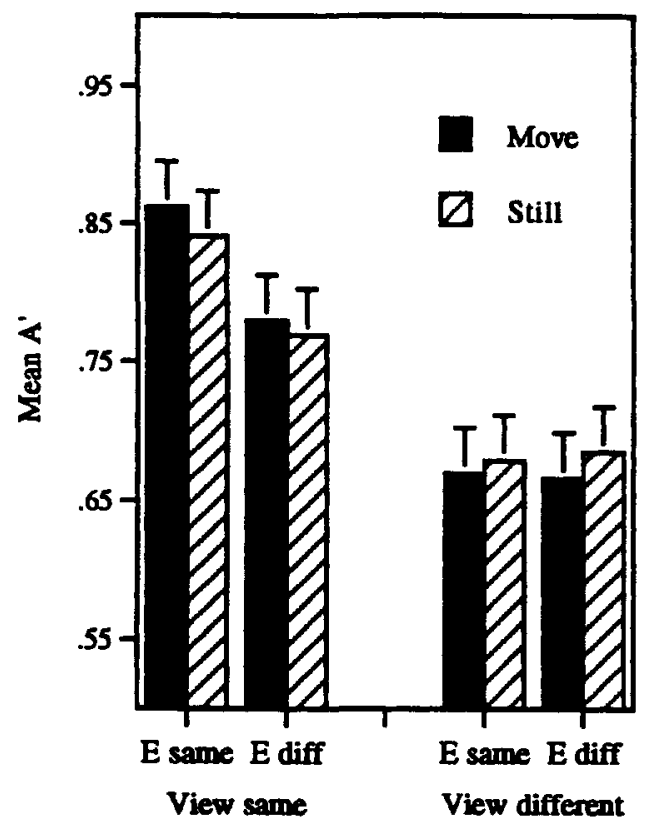

Figure 2. Mean $A^{\prime}$ in each condition of Experiment $1 B$ (dynamic test). The error bars show the $95 \%$ confidence interval for the within-subjects comparison between presentation conditions (move vs. still) as a function of expression ( $E$, same or different) and viewpoint (same or different).

there is a rotation within the horizontal plane. When the head nods, the viewpoint is FF throughout the gesture, and there is a transition within the vertical plane. In this experiment, different groups of participants studied faces showing the nod or the shake gesture. The plane of gesture shown at test was either the same or different from that studied (corresponding to a change in viewpoint). The third factor allowed us to explore differences between the nod and shake gestures themselves. The factor examined was gesture at test, rather than the gesture studied, since distractor faces were seen only at test; this allowed us to investigate differences between gestures on hits, FPs, and their combination. Examining the effects of test gesture alongside those of changing or maintaining plane allows differences between nod and shake gestures in the study phase to be inferred. In Experiment $2 \mathrm{~A}$, targets were tested using single static images; in Experiment 2B, they were tested with moving sequences.

\section{Method}

Participants. These were 32 staff or students in Experiment $2 \mathrm{~A}$ and 32 in $2 \mathrm{~B}$, all between 17 and 37 years (equal numbers of males and females in 2A, 15 males and 17 females in 2B). In each experiment, there were 8 participants per condition.

Materials. For the moving versus static presentation, the study phase for the nod gesture comprised a series of three frames shown from an $\mathrm{FF}$ plane/head-on perspective; for the moving sequences, each series was played seven times in the correct running order. There were two blank, black frames at the beginning and end of each run of 21 . The static nodding sequence was shown in a method similar to that used in the static study phase in the first two experiments, with each individual frame being repeated seven times in succession; two blank, black frames separated each "block." The order in which these blocks were shown was designed to minimize any potential coherence of transition between them, so that the face seemed to "jump" between each constituent element of its sequence.

The shake gesture was also portrayed by a series of three frames. Instead of a full sweep (either left to right, or right to left), the head was shown carrying out only half of the sweep - that is, from side to center. Six independent observers judged that this was a realistic gesture (when shown using Xrastool animation). The moving and static shake sequences were produced in the same way as their nod counterparts. For the shake gesture, targets were randomly allocated a left-to-center, or a right-to-center sweep.

In the test phases, participants in Experiment 2A were tested using the Superlab application, which displayed a single static frame. The frame selected for targets and distractors was the apex of the appropriate gesture (upward for the nod and the corresponding sideview for the shake).

For Experiment $2 B$, the methodology used was as in Experiment $1 B$, in which verbal recognition decisions were based on animated moving test sequences, shown using two Xrastool movies on the Sun Workstation.

Design. The $2 \times 2 \times 2$ factorial design of this experiment enabled examination of the variable of presentation format, either moving or static (within subjects); plane, either same or changed between learning and test (between subjects); and gesture at test, either nod or shake (between subjects). In Condition 1, same plane-shake, participants studied the targets performing a shake gesture sequence and were tested with an image (Experiment $2 \mathrm{~A}$ ) or sequence (2B) from the shake gesture. In Condition 2, same plane-nod, participants studied targets performing the nod gesture and were tested with an image/sequence from the nod gesture. In Condition 3 , different plane-shake, participants studied targets performing the nod gesture and were tested with an image/sequence of the shake gesture. In Condition 4, different plane-nod, participants studied targets performing the shake gesture and were tested with an image/sequence from the nod gesture.

Procedure. The procedure was the same as in Experiments $1 \mathrm{~A}$ and $1 \mathrm{~B}$.

\section{Results of Experiment 2A}

The mean hit rate across all conditions was $58 \%$; the FP rate was $26 \%$. Figure 3 plots the mean $A^{\prime}$ in each condition of interest. In this analysis, discrimination of old from new faces was not significantly worse when plane was changed $[F(1,28)=2.24, p>.1]$. The $\mathrm{A}^{\prime}$ analysis showed a significant effect of condition, with discrimination better when static faces had been studied $[F(1,28)=$ $5.21, p<.05]$. No interactions reached significance (all $p s>.1)$.

ANOVA of hit rates, however, revealed the effects of changing plane $[F(1,28)=5.41, p<.05$; but $p=.068$ by items] and of presentation condition to be significant. Analysis of FPs showed no significant effects (see Table 3 for hit and FP rates in each condition).

\section{Results of Experiment 2B}

Performance in Experiment 2B was a little higher overall, with an average hit rate across all conditions of $75 \%$ and an FP rate of $23 \%$. Figure 4 shows the mean $A^{\prime}$ in each condition. ANOVA of $A^{\prime}$ here showed no significant main effects or interactions (all $p \mathrm{~s}>.1$ ).

However, there was again a significant main effect in the analysis of hit rates for the plane variable $[F(1,28)=$ 
Table 3

Mean Percentages Hits and False Positives for Plane (Same/Different) and Test Gesture (Nod/Shake), With Standard Deviations, for Experiment 2A

\begin{tabular}{|c|c|c|c|c|c|c|c|c|c|c|c|c|c|c|c|}
\hline \multicolumn{16}{|c|}{ Presentation Format } \\
\hline \multicolumn{8}{|c|}{ Moving } & \multicolumn{8}{|c|}{ Static } \\
\hline \multicolumn{4}{|c|}{ Same } & \multicolumn{4}{|c|}{ Diff } & \multicolumn{4}{|c|}{ Same } & \multicolumn{4}{|c|}{ Diff } \\
\hline \multicolumn{2}{|c|}{ Nod } & \multicolumn{2}{|c|}{ Shake } & \multicolumn{2}{|c|}{ Nod } & \multicolumn{2}{|c|}{ Shake } & \multicolumn{2}{|c|}{ Nod } & \multicolumn{2}{|c|}{ Shake } & \multicolumn{2}{|c|}{ Nod } & \multicolumn{2}{|c|}{ Shake } \\
\hline$M$ & $S D$ & $M$ & $S D$ & $M$ & $S D$ & $M$ & $S D$ & $M$ & $S D$ & $M$ & $S D$ & $M$ & $S D$ & $M$ & $S D$ \\
\hline \multicolumn{16}{|c|}{ Hits } \\
\hline 65 & 23 & 55 & 26 & 48 & 24 & 38 & 23 & 72 & 21 & 73 & 15 & 58 & 29 & 60 & 26 \\
\hline \multicolumn{16}{|c|}{ False Positives } \\
\hline 20 & 7 & 29 & 16 & 31 & 14 & 23 & 14 & 20 & 7 & 29 & 16 & 31 & 14 & 23 & 14 \\
\hline
\end{tabular}

$5.82, p<.05]$, with more faces recognized when tested in the same plane. The effect of presentation format also approached significance, with a trend toward an advantage for a static learning sequence [hits $\times$ participants, $F(1,28)=$ $4.06, p=.054$, but $\times$ items, $F(1,9)=2.92, p>0.1]$.

The only other significant effect that arose in any of the analyses was an interaction in the items analysis of hits between the plane variable and the test gesture $[F(1,9)=10.56, p<.01]$, reflecting the tendency for the drop in performance to be greater when the plane is changed from shaking at study to nodding at test than when the plane is changed from nodding at study to shaking at test.

There were no significant effects in analyses of false positive rates (see Table 4 for mean hit and false positive rates in each condition).

\section{Discussion}

In contrast to previous experiments, Experiment 2A did show a significant main effect of presentation format, but the advantage was in favor of recognizing those faces learned from static sequences. Contrary to the experimental hypothesis, we find that learning a face from a moving sequence actually confers a disadvantage. Experiment $2 \mathrm{~B}$, which used dynamic test sequences, removed this disadvantage but did not reverse it, and the trend still favored static presentation.

In both parts of the experiment, participants were again somewhat better at recognizing targets within the same plane, but these differences were significant only in the analyses of hits, and not in the analysis of $A^{\prime}$. Effects of viewpoint change, which were strong in Experiments $1 \mathrm{~A}$ and $1 \mathrm{~B}$, were considerably reduced by study of changing viewpoints. There appeared to be no major differences between the nod (based on FF, vertical rotations) and the shake (based on TQ, horizontal rotations) gestures.

In order to compare more directly the effects of presentation and test format and changing view, we conducted a combined analysis of the $\mathrm{A}^{\prime}$ results from all four experiments. This combined analysis also had the advantage of adding power, since, as indicated in Tables 1-4 and Figures 1-4, there was considerable variability within each individual experiment.

\section{Combined Analysis}

A post hoc $2 \times 2 \times 2 \times 2$ ANOVA examined participants' $A^{\prime}$ scores across all four experiments to examine the overall effects of presentation format at study (static vs. dynamic), format at test (static vs. dynamic), viewpoint (same or changed), and type of gesture (internal expressive or global, rigid movements). This analysis collapsed across levels of the third variable in each experiment (i.e., the particular expression or test gesture used).

This ANOVA showed a significant effect of presentation format, with the trend favoring static presentation $[F(1,128)=5.3, p<.05]$. There was a highly significant main effect of viewpoint, with performance worse when view was changed $[F(1,128)=29.85, p<.001]$, and a main effect of gesture, with rigid head movements (Ex-

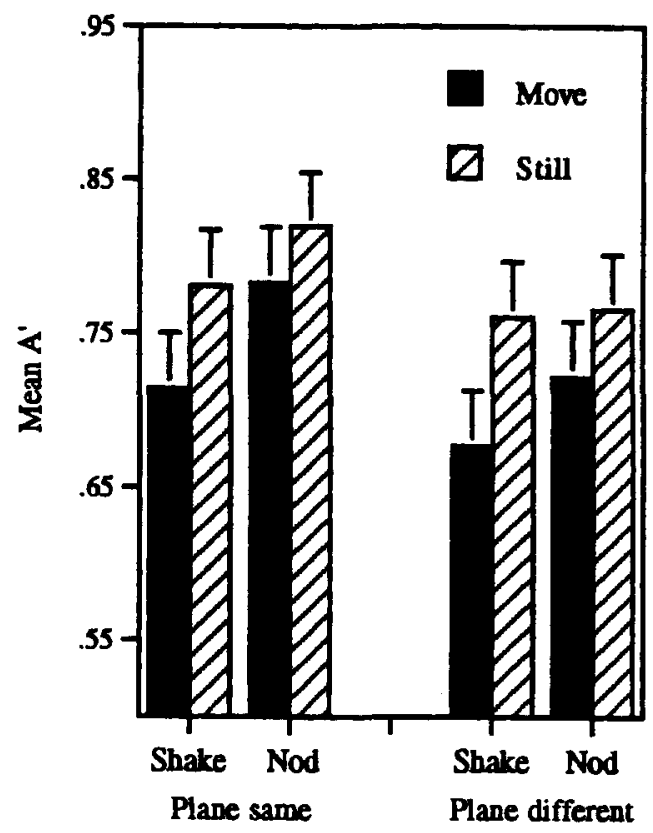

Figure 3. Mean $A^{\prime}$ in each condition of Experiment $2 A$ (static test). The error bars show the $95 \%$ confidence interval for the within-subjects comparison between presentation conditions (move vs. still) as a function of gesture at test (shake vs. nod) and plane of testing (same as or different from presentation). 
Table 4

Mean Percentages Hits and False Positives, for Plane (Same/Different) and Test Gesture (Nod, Shake), With Standard Deviations, for Experiment 2B

\begin{tabular}{|c|c|c|c|c|c|c|c|c|c|c|c|c|c|c|c|}
\hline \multicolumn{16}{|c|}{ Presentation Format } \\
\hline \multicolumn{8}{|c|}{ Moving } & \multicolumn{8}{|c|}{ Static } \\
\hline \multicolumn{4}{|c|}{ Same } & \multicolumn{4}{|c|}{ Diff } & \multicolumn{4}{|c|}{ Same } & \multicolumn{4}{|c|}{ Diff } \\
\hline \multicolumn{2}{|c|}{ Nod } & \multicolumn{2}{|c|}{ Shake } & \multicolumn{2}{|c|}{ Nod } & \multicolumn{2}{|c|}{ Shake } & \multicolumn{2}{|c|}{ Nod } & \multicolumn{2}{|c|}{ Shake } & \multicolumn{2}{|c|}{ Nod } & \multicolumn{2}{|c|}{ Shake } \\
\hline$M$ & $S D$ & $M$ & $S D$ & $M$ & $S D$ & $M$ & $S D$ & $M$ & $S D$ & $M$ & $S D$ & $M$ & $S D$ & $M$ & $S D$ \\
\hline \multicolumn{16}{|c|}{ Hits } \\
\hline 78 & 23 & 83 & 17 & 50 & 21 & 73 & 24 & 80 & 21 & 88 & 15 & 70 & 21 & 80 & 19 \\
\hline \multicolumn{16}{|c|}{ False Positives } \\
\hline 25 & 20 & 22 & 17 & 25 & 13 & 20 & 12 & 25 & 20 & 22 & 17 & 25 & 13 & 20 & 12 \\
\hline
\end{tabular}

periments $2 \mathrm{~A}$ and $\mathrm{B}$ ) leading to higher scores than expressive ones (Experiments $1 \mathrm{~A}$ and $\mathrm{B}$ ) $[F(1,128)=5.64, p<$ $.05]$. These main effects were qualified by a significant interaction between viewpoint and gesture $[F(1,128)=$ $5.84, p<.05]$. Effects of viewpoint change were greater when expressive changes were studied (Experiments $1 \mathrm{~A}$ and $B$ ) than when rigid changes were studied (Experiments $2 A$ and $B$ ). Figure 5 plots this interaction, showing separately the means for moving and static study conditions. Finally, the combined analysis showed a nearly significant effect of test format, with dynamic test conditions (Experiments $1 \mathrm{~B}$ and $2 \mathrm{~B}$ ) producing higher $\mathrm{A}^{\prime}$ scores $[F(1,128)=3.48, p=.064]$.

\section{GENERAL DISCUSSION}

The findings from this series of experiments suggest that motion per se is not an important factor in establishing representations for unfamiliar faces. Throughout the experimental series, the trends favored study from a series of static instances. In the introduction, we described experiments by Pike et al. (1997), who did find a significant advantage for using movement (a video sequence) in the study of unfamiliar faces shown in rigid rotation in depth, and who tested using a single (static) photograph. Pike et al. suggested that movement helped in building up a 3-D representation, based additionally on analysis of shape from shading. Experiments 2A and 2B presented in this paper used a similar type of gesture (the shake sequence), but unlike Pike et al., we found advantages (significant in Experiment 2A) for a static learning phase. One possible reason for the discrepancy is that Pike et al.'s results may have arisen from the increased information content of their moving compared with static conditions, though this seems unlikely given that in some experiments as many as 10 static images were shown to yield decreased performance compared with the moving condition. Another possibility is that differences between the results obtained by Pike et al.'s results and the present findings arose because in their studies lighting conditions were changed between study and test, whereas in the present study they were maintained. Observers find it very difficult to match images of faces shown in different lighting conditions (Hill \& Bruce, 1996), and it is possible that 3-D structure-from-motion information may play a more prominent role in such circumstances.

As well as equating the static information content of moving and static presentation conditions, in our studies we explored whether movement enhanced representations established when expressive movements were made to compare these effects with those of rigid movements. Again we found no benefit for studying images of faces shown in expressive movements compared with the same static information presented in a nonanimated sequence.

One disadvantage of the methodology we employed, however, is that in order to equate the information content of the static and dynamic study sequences, we had to utilize very parsimonious displays (particularly the use of only three frames showing the rotational changes in Experiments $2 \mathrm{~A}$ and $2 \mathrm{~B}$ ), which may have encouraged a

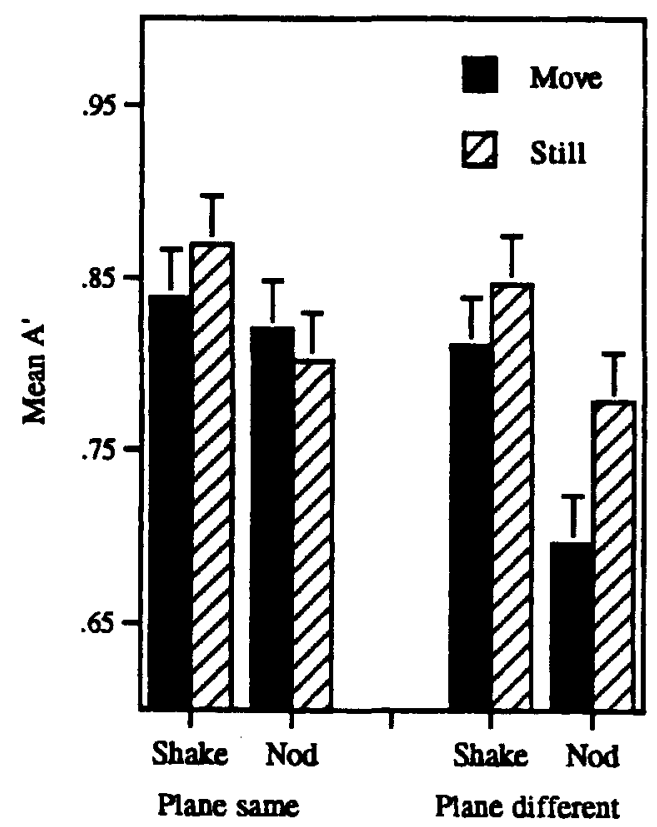

Figure 4. Mean $A^{\prime}$ in each condition of Experiment 2B (dynamic test). The error bars show the $95 \%$ confidence interval for the within-subjects comparison between presentation conditions (move vs. still) as a function of gesture at test (shake vs. nod) and plane of testing (same as or different from presentation). 


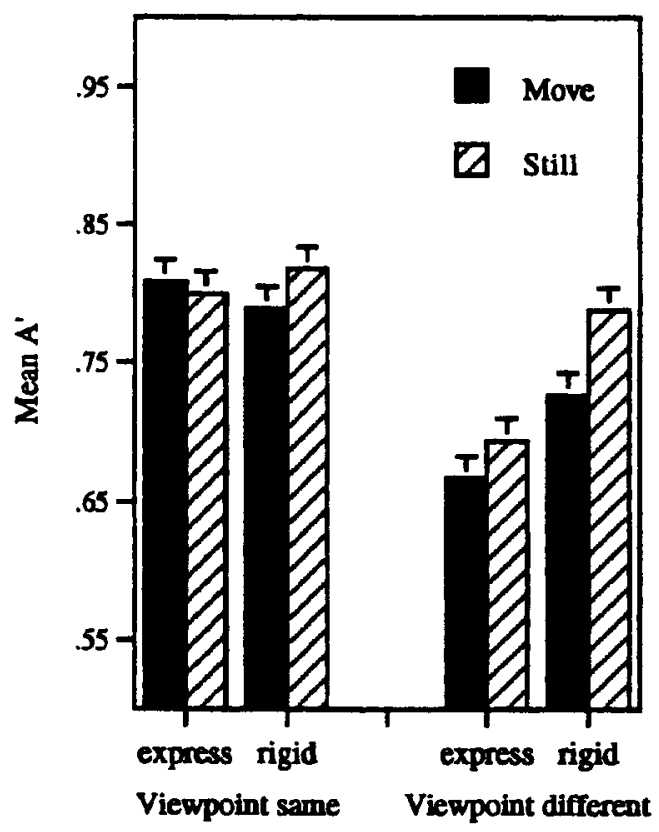

Figure 5. Mean $A^{\prime}$ in the combined analysis of all four experiments, showing how effects of viewpoint are moderated by gesture, for static and moving presentation. The error bars show the $95 \%$ confidence interval for the within-subjects comparison between presentation conditions (move vs. still).

"pictorial" or "event-specific" encoding. However, any such pictorial effects might be expected to lead to significantly better memory at test when any of those study conditions were recreated. If that were the case, it is not clear why, for example, the effect of presentation format was not significantly reversed in favor of motion in Experiment $2 \mathrm{~B}$, in which both phases of the experiment featured dynamic sequences. Nonetheless, it should be acknowledged that attempts to equate static and dynamic information content while preserving realistic motion sequences have their own limitations.

All experiments showed some decrement in hit rates when the faces were tested from a different viewpoint ("view" in Experiments $1 \mathrm{~A}$ and 1B; "plane" in Experiments $2 \mathrm{~A}$ and $2 \mathrm{~B}$ ), though significant effects in the $\mathrm{A}^{\prime}$ analyses were confined to Experiments $1 \mathrm{~A}$ and $1 \mathrm{~B}$. Effects of viewpoint change were greater when the dynamic changes that had been studied were of facial expressions rather than rotations of the whole head in depth. This suggests that studying a sequence of varying viewpoints does provide some information to help generalize to a new viewpoint, even when this involves an image shown in a different plane from that of the movements studied (Experiments $2 \mathrm{~A}$ and $2 \mathrm{~B}$ ). The experimental hypothesis postulated that a face learned via a moving sequence should provide additional information (over and above that given in a sequence of static instances) about the 3-D structure of the face, and thus should aid recognition of faces in changed viewpoints, yet in none of our experi- ments was there any indication that studying moving sequences ameliorated effects of changing viewpoint. When study conditions most favor the construction of a 3-D representation of the face, by showing rotations of the head in depth (i.e., in the last pair of experiments), generalization to a different plane/viewpoint was indeed enhanced, but was not further helped by having seen the faces move.

Our studies do not rule out any role of dynamic information in face recognition. We found a trend in the combined $\mathrm{A}^{\prime}$ analysis toward an advantage for testing with moving sequences, and this finding may be compared with recent results using familiar faces. For example, Knight and Johnston (1997) have used image manipulations (such as inversion and photographic negative), which are known to have detrimental effects on familiar face identification, in order to tease out the differential contributions of moving and static information. They found that significantly more famous faces were recognized when shown in negative moving sequences, rather than as a single negative, static frame. One possible explanation is that increased information in the moving test conditions is responsible for the effects, since information was not equated in the test phases in our own or in Knight and Johnston's experiments. Thus movement in the test phase may provide more retrieval cues than a single static viewpoint.

Nonetheless, it is also possible that as faces become familiar, the way that a particular face moves may become incorporated in the representation used to recognize it. We may encode characteristic movement patterns alongside representations of the face shape (e.g., Dame Edna Everidge's exaggerated lip movements). If the task of recognizing famous faces is made more difficult by experimental manipulations (such as inversion, negation, or filtering), it may be that these movements aid our recognition, and not the features themselves. These dynamic events cannot be encapsulated by sampling mere static instances. Indeed, this aspect of idiosyncratic movement may underlie the art of impersonation. The success of impersonators may depend upon the extent to which they are able to manipulate their own face configurations to reproduce, or even exaggerate, the imitated character's typical dynamic facial gestures. Future research using both familiar and unfamiliar faces presented in dynamic and static test conditions will be needed to explore these possibilities.

\section{REFERENCES}

Bartlett, J. C., \& Leslie, J. E. (1986). Aging and memory for faces versus single views of faces. Memory \& Cognition, 14, 371-381.

Bassili, J. N. (1978). Facial motion in the perception of faces and of emotional expression. Journal of Experimental Psychology: Human Perception \& Performance, 4, 373-379.

Biederman, I. (1987). Recognition by components: A theory of human image understanding. Psychological Review, 94, 115-145

BruCE, V. (1982). Changing faces: Visual and non-visual coding processes in face recognition. British Journal of Psychology, 73, 105-1 16. 
BRUCE, V. (1994). Stability from variation: The case of face recognition: The M. D. Vernon Memorial Lecture. Quarterly Journal of Experimental Psychology, 47A, 5-28.

BRUCE, V., \& VALENTINE, T. (1988). When a nod's as good as a wink. The role of dynamic information in facial recognition. In $\mathbf{M}$. M. Gruneberg \& E. Morris (Eds.), Practical aspects of memory: Current research and issues (pp. 169-174). Hillsdale, NJ: Erlbaum.

BRUCE, V., \& YouNG, A. W. (1986). Understanding face recognition. British Journal of Psychology, 77, 305-327.

Cabeza, R., Bruce ,V., Kato, T., \& ODA, M. (in press). The prototype effect in face recognition: Extension and limits. Memory \& Cognition.

Davies, G. M., Ellis, H. D., \& Shepherd, J. W. (1978). Face recognition as a function of mode of representation. Journal of Applied Psychology, 63, 180-187.

Hill, H., \& BRUCE, V. (1996). Effects of lighting on matching facia surfaces. Journal of Experimental Psychology: Human Perception \& Performance, 22, 986-1004.

KLATZKY, R. L., \& FORREST, F. H. (1984). Recognizing familiar and unfamiliar faces. Memory \& Cognition, 12, 60-70

KNight, B., \& Johnston, A. (1997). The role of movement in face recognition. Visual Cognition, 4, 265-274.

LofTus, G. R., \& MAsson, M. E. J. (1994). Using confidence intervals in within-subject designs. Psychonomic Bulletin \& Review, 1, 476-490.

MARR, D., \& NishihaRA, H. K. (1978). Representation and recognition of the spatial organisation of three-dimensional shapes. Proceedings of the Royal Society of London: Series B, 200, 269-294.

Perrett, D. I., Smith, D. D., Potter, P. A. J., Mistlin, A. J., Head, A. S., Milner, A. D., \& Jeeves, M. A. (1985). Visual cells in the temporal cortex sensitive to face view and gaze direction. Proceed ings of the Royal Society of London: Series B, 223, 293-317.
Pike, G. E., Kemp, R. I., Towell, N. A., \& Phillips, K. C. (1997), Recognizing moving faces: The relative contribution of motion and perspective view information. Visual Cognition, 4, 409-438.

SChIFF, W., Banka, L., \& de Bordes Galai, G. (1986). Recognizing people seen in events via dynamic mug-shots. American Journal of Psychology, 99, 219-231.

Shapiro, P. N., \& Penrod, S. (1986). Meta-analysis of facial identification studies, Psychological Bulletin, 100, 139-156.

Shepherd, J. W., Ellis, H. D., \& Davies, G. H. (1982). Identification evidence: A psychological evaluation. Aberdeen: University of Aberdeen Press.

Snodgrass, J. G., \& Corwin, J. (1988). Pragmatics of measuring recognition memory-Applications to dementia and amnesia. Journal of Experimental Psychology: General, 117, 34-50.

TARR, M. J., \& BulThOFF, H. H. (1995). Is human object recognition better described by geon-structural-descriptions, or by multiple views? Journal of Experimental Psychology: Human Perception \& Performance, 21, 1494-1505.

Tulving, E., \& Thomson, D. (1983). Encoding specificity and retrieval processes in memory. Psychological Review, 80, 352-372.

Ullman, S. (1979). The interpretation of visual motion. Cambridge, MA: MIT Press.

VOKEY, J. R., \& READ, J. D. (1992). Familiarity, memorability, and the effect of typicality on the recognition of faces. Memory \& Cognition, 20, 291-302

(Manuscript received March 21, 1996, revision accepted for publication March 13, 1997.) 